\title{
Pja2 Inhibits Wnt/ $\beta$-catenin Signaling by Reducing the Level of TCF/LEF1
}

\author{
Yonghee Song ${ }^{1}$, Somyung Lee ${ }^{1}$, Jeong-Rae Kim², Eek-hoon Jho ${ }^{1}$ \\ ${ }^{1}$ Department of Life Science, University of Seoul, Seoul, Korea \\ ${ }^{2}$ Department of Mathematics, University of Seoul, Seoul, Korea
}

Ubiquitination of proteins plays an essential role in various cellular processes, including protein degradation, DNA repair, and cell signaling pathways. Previous studies have shown that protein ubiquitination is implicated in regulating pluripotency as well as fate determination of stem cells. To identify how protein ubiquitination affects differentiation of embryonic stem cells, we analyzed microarray data, which are available in the public domain, of E3 ligases and deubiquitinases whose levels changed during stem cell differentiation. Expression of pja2, a member of the RING-type E3 ligase family, was up-regulated during differentiation of stem cells. Wnt $\beta$-catenin signaling is one of the most important signaling pathways for regulation of the self-renewal and differentiation of embryonic stem cells. Pja2 was shown to bind to TCF/LEF1, which are transcriptional factors for $\mathrm{Wnt} / \beta$-catenin signaling, and regulate protein levels by ubiquitination, leading to down-regulation of Wnt signaling activity. Based on these results, we suggest that E3 ligase Pja2 regulates stem cell differentiation by controlling the level of TCF/LEF1 by ubiquitination.

Keywords: Pja2, E3 ligase, Wnt, TCF/LEF1, Embryonic stem cell, Differentiation

\section{Introduction}

Embryonic stem cells (ESCs) have pluripotency and differentiation capacity, which are useful for various clinical applications (1, 2). Regulation of stemness and differentiation of ESCs have been major goals of researchers for decades. Treatment with small molecules or recombinant proteins as well as overexpression or knockdown of proteins involved in various signaling pathways have been

Received April 5, 2018, Revised June 13, 2018, Accepted June 14, 2018, Published online August 31, 2018

Correspondence to Eek-hoon Jho

Department of Life Science, University of Seoul, 163, Seoulsiripdae-ro, Dongdaemun-gu, Seoul 02504, Korea

Tel: +82-2-6490-2671, Fax: +82-2-6490-2664

E-mail: ej70@uos.ac.kr

(c) This is an open-access article distributed under the terms of the Creative Commons Attribution Non-Commercial License (http://creativecommons.org/ licenses/by-nc/4.0/), which permits unrestricted non-commercial use, distribution, and reproduction in any medium, provided the original work is properly cited.

Copyright (c) 2018 by the Korean Society for Stem Cell Research utilized for regulation of ESC differentiation (3-7). For differentiation of ESCs, expression levels of numerous proteins must be sufficiently altered in order to overcome the differentiation barrier at an early stage. To overcome this barrier, we hypothesized that levels of proteins specifically related to maintenance of stemness should be down-regulated. E3 ligases and deubiquitinases (DUBs) play crucial roles in the regulation of protein levels by proteasomal degradation (8). We analyzed publically available microarray databases and searched for E3 ligases whose levels were altered during differentiation.

$\mathrm{Pja} 2$ is a RING domain E3-ubiquitin ligase that is expressed in most mammalian tissues. Originally, pja2 was named as Neurodapl due to its function related to neurodegeneration $(9,10)$. Pja2 participates in several signaling pathways as a protein regulatory factor. Upon cyclic AMP stimulation, pja2 ubiquitinates the regulatory $(\mathrm{R})$ subunit of PKA, which plays an inhibitory role against PKA activity. Induced proteasomal degradation of R-subunit by pja2 promotes cAMP-mediated target gene expression (11). Pja2 also regulates hippo signaling via ubiquitina- 
tion-dependent proteolytic degradation of mobl, which causes inhibition of Lats1/2. Inhibition of Lats1/2 enhances expression of yap target genes. Finally, overexpressed pja2 induces cell proliferation in human glioblastoma (12). However, the function of pja2 in stem cell differentiation remains elusive.

Wnt signaling has been described as a regulator of ESCs, although its role in ESC differentiation is still debatable. Activation of Wnt signaling is required for various types of stem cell maintenance and differentiation (13, 14). However, according to several reports, transcriptional activity of $\beta$-catenin does not play a role in mouse ESC differentiation (15). Activated Wnt signaling induces nuclear translocation of $\beta$-catenin, which proceeds to interact with the transcription factors TCF/LEF1 and enhance expression of their target genes. The levels of TCF/LEF1 are regulated at multiple stages from transcriptional regulation to post-translational modification (16-19).

Here, we report an inverse correlation between the levels of pja2 and TCF/LEF1 during differentiation of mouse ESCs and provide an evidence that pja2 is an E3 ligase for TCF/LEF1. Our data suggest that pja2 interacts with TCF/LEF1 and enhances ubiquitin-mediated proteasomal degradation of TCF/LEF1.

\section{Materials and Methods}

\section{Culture of cells and Transfection}

Mouse E14 embryonic stem cells (mESCs) and human embryonic kidney HEK293T cells were used for the experiments. mESCs were cultured on tissue culture plates (Sigma) pre-coated with $0.2 \%$ gelatin at $37^{\circ} \mathrm{C}$ in a $5 \% \mathrm{CO}_{2}$ incubator. Cell culture media are consisted of $15 \%$ fetal bovine serum (FBS, Biowest), $1 \mathrm{mM}$ sodium pyruvate (GIBCO), 100X non-essential amino acid (Corning), $0.1 \mathrm{mM} \beta$-mercaptoethanol (GIBCO), 100X GlutaMAX (GIBCO), Tylosine (Sigma), and 1,000 U/mL of LIF (conditioned media). LIF-containing media were produced from Cos7 cells transfected with LIF cDNA by Lipofectamine. Subculture was performed by $0.01 \%$ trypsin treatment for $3 \mathrm{~min}$ in a $37^{\circ} \mathrm{C}$ incubator. After incubation, trypsin was neutralized by FBS-containing media, and mESCs were collected by centrifugation. Subsequently, mESCs were plated on a pre-coated culture dish. HEK293T cells were maintained on a SPL tissue culture plate with Dulbecco's Modified Eagle's Medium (DMEM). Media contained 10\% fetal bovine serum (FBS, Gibco) and 1X antibiotics (anti-anti, Gibco). Subculture was performed with $0.05 \%$ trypsin. Cells were re-suspended with FBS-containing media for neutralization and re-plated on a tissue culture plate. Calcium phosphate method was used for plasmid DNA transfection.

\section{mESC differentiation}

Differentiation of mESC was induced by removing LIF from culture media. The media for mESC were substituted with media without LIF 1 day after subculture. Every 2 days, media were exchanged with fresh media.

\section{cDNA synthesis and quantitative PCR}

mRNAs from E14 and HEK293T cells were isolated by TRIzol (Thermo Fisher) according to the manufacturer's protocol. RTase mastermix (TOYOBO) was used for cDNA synthesis. qPCR was performed by using CFX Connect $^{\mathrm{TM}}$ real-time PCR (BIO-RAD) with SYBR qPCR mix (TOYOBO).

\section{Immunoprecipitation and immunoblotting}

Cell lysates were prepared in lysis buffer $(20 \mathrm{mM}$ Tris- $\mathrm{HCl} \mathrm{pH} 7.5,150 \mathrm{mM} \mathrm{NaCl}, 1 \%$ Triton X-100, $1 \mathrm{mM}$ EDTA, $1 \mathrm{mM}$ EGTA, $2.5 \mathrm{mM}$ sodium pyrophosphate, 1 $\mathrm{mM} \beta$-glycerophosphate, and $1 \mathrm{mM}$ sodium orthovanadate). Lysates were immunoprecipitated overnight using the indicated antibody. Subsequently, immunoprecipitates were pulled down by protein $\mathrm{A} / \mathrm{G}$ plus agarose beads (Santa Cruz). After elution by boiling, proteins were separated by SDS-PAGE and transferred onto a PVDF membrane. Each primary antibody was treated overnight with $5 \%$ BSA or $5 \%$ skim milk at $4^{\circ} \mathrm{C}$. Mouse and rabbit secondary antibodies were treated for 2 hours at room temperature. Proteins were detected by MicroChemi 4.2 (DNR Bio-Imaging System). The following antibodies were used: anti-pja2 (Bethyl), anti-TCF1 (cell signaling), anti-LEF1 (cell signaling), anti-HA (Santa Cruz), anti-Myc (Abm), anti-Oct4 (Santa Cruz), and anti-Flag (Sigma).

\section{Luciferase assay}

TOP-luciferase reporter plasmid, pRL-TK-Renilla, and the plasmids indicated in the figure were transfected into HEK293T cells plated on a 12-well plate. At 16 hours after transfection, luciferase assay was performed according to the manufacturer's protocol (Promega, \#E1960), and its activity was measured by a GLOMAX 20/20 luminometer (Promega). Transfection efficiency was normalized by pRL-TK-Renilla-mediated luciferase activity. 


\section{Results}

\section{Increased level of Ring-finger domain E3 ligase pja2} during differentiation of mouse embryonic stem cells

We analyzed publically available microarray databases and searched for E3 ligases whose levels increased during differentiation. pja2 showed appropriate expression patterns in our analysis. To assess the validity of the observed increased in pja2 RNA expression in the microarray analysis, we differentiated mESCs by removal of LIF and performed quantitative real-time PCR. As shown in Figure 1A, pja2 RNA expression increased during mESC differentiation (Fig. 1A). Western blot showed that pja2 protein levels increased consistent with its RNA expression, while the level of Oct4, a marker for undifferentiated mESC, was decreased (Fig. 1B). These data indicate that pja2 mRNA and protein levels increased during mESC differentiation.

\section{pja2 negatively regulates $\mathrm{Wnt} / \beta$-catenin signaling}

Wnt signaling is known to play critical roles both in the maintenance of stemness as well as differentiation of ESCs. Reduced Wnt $\beta$-catenin signaling initiates differentiation of mESCs. Thus, we hypothesized that Wnt signaling components can be putative targets of pja2. We tested this hypothesis by performing Wnt reporter luciferase assay. Ectopic expression of HA-tagged pja2 reduced relative luciferase activity under control (L-CM) or Wnt3a-conditioned media (Wnt3a-CM) conditions (Fig. 2A). To elucidate which components of the Wnt signaling pathway are targeted by pja2, additional reporter assays were performed. Each Wnt signaling activating compo- nent, including LRP $\triangle \mathrm{N}$ (constitutive active LRP), Dvl, $\beta$-catenin, TCF1, LEF1, and VP16-LEF $\Delta \mathrm{N}$ (constitutive active LEF1), was overexpressed with pja2. All luciferase activities induced by Wnt activating components were reduced by pja2 overexpression, and even VP16-LEF $\Delta \mathrm{N}$-induced signaling was down-regulated (Fig. 2B). These results suggest that pja2 inhibited $\mathrm{Wnt} / \beta$-catenin signaling at the same level or downstream of TCF/LEF1.

\section{pja2 interacts with and reduces the level of TCF/LEF1}

Our data shown in Figure 2 suggest that TCF and/or LEF are putative targets of pja2. Thus, we examined the protein dynamics of pja2 and TCF/LEF during mESC differentiation. Results from the Western blot show that the levels of TCF1, LEF1, and Oct4 decreased during mESC differentiation. Interestingly, the level of pja2 showed an inverse correlation during mESC differentiation (Fig. 3). To regulate target proteins, E3 ligase should interact with its substrates. We then investigated the interaction between pja2 and TCF/LEF1. Immunoprecipitation analysis found that pja2 and TCF/LEF1 clearly interacted with each other (Fig. 4A). However, the inverse correlation protein dynamics and protein interaction data do not prove that pja2 inhibited the level of TCF/LEF1. Knockdown of human $P \mathcal{F A} 2$ by siRNAs increased both TCF1 and LEF1 levels in HEK293T cells, whereas co-transfection with pja2 plasmid, which contains a mutated sequence resistant to $P f A 2$-specific siRNAs, reduced levels of TCF1 and LEF1 (Fig. 4B). Since ectopic expression of pja2 reduced the levels of TCF1 and LEF1, we tested whether or not pja2 could enhance ubiquitination of TCF1 and LEF1. MYC-tagged pja2 was transfected
A

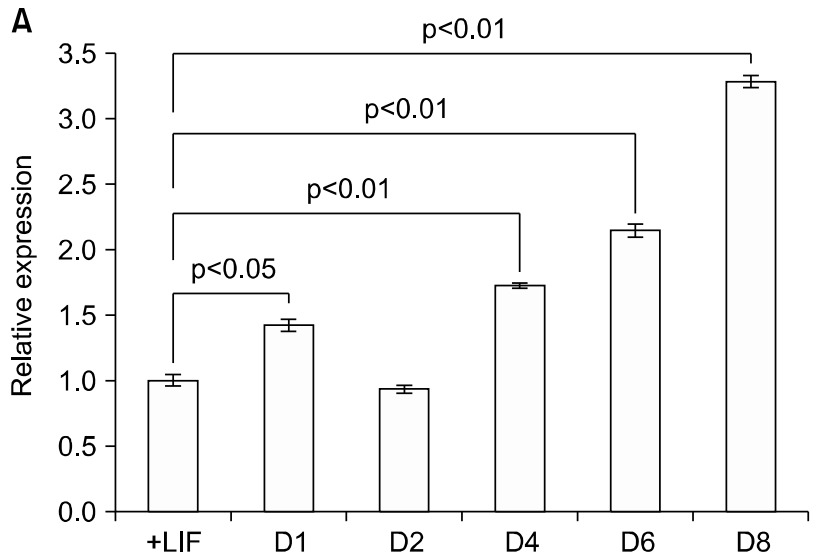

B

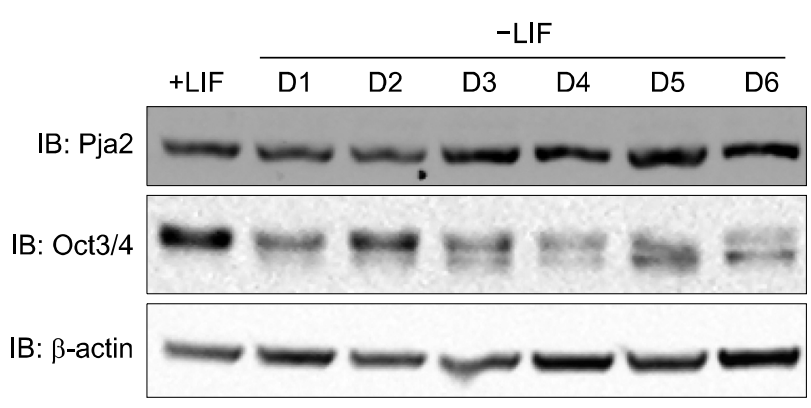

Fig. 1. Levels of pja $2 \mathrm{mRNA}$ and protein increase during mESC differentiation. (A) qPCR analyses for pja2 mRNA expression during mESC differentiation. Total RNAs were isolated at the indicated times. (B) Lysates were obtained at the indicated times, and the levels of proteins were detected by Western blotting using the indicated antibodies. Differentiation of mESCs was induced by withdrawal of LIF from culture media. 
A

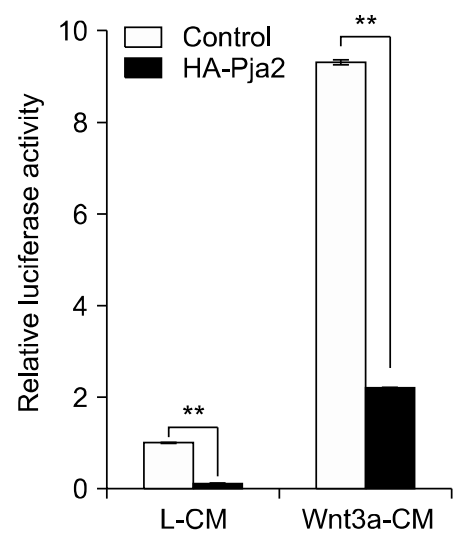

B

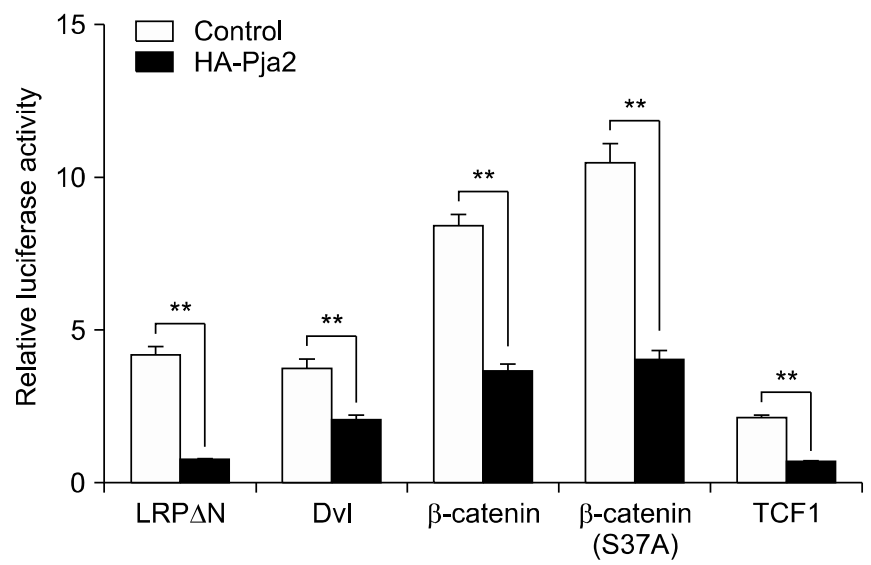

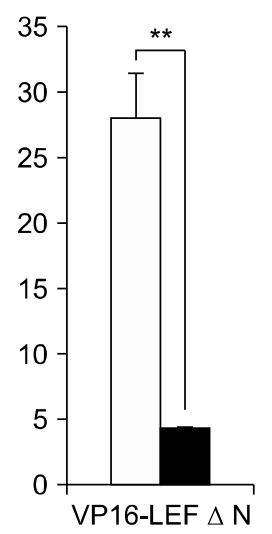

Fig. 2. Pja2 inhibits Wnt/ $\beta$-catenin signaling. (A) Pja2 inhibits Wnt 3a-mediated reporter activity. TOP-Flash Wnt reporter construct was transfected with HA-pja2. Reporter activity was analyzed upon treatment with control and Wnt 3a-conditioned media. (B) Pja2 inhibits activator of Wnt signaling-mediated reporter activity. Reporter activity was measured after transfection with the indicated plasmids into HEK293T cells. ${ }^{* *} \mathrm{p}<0.01$.

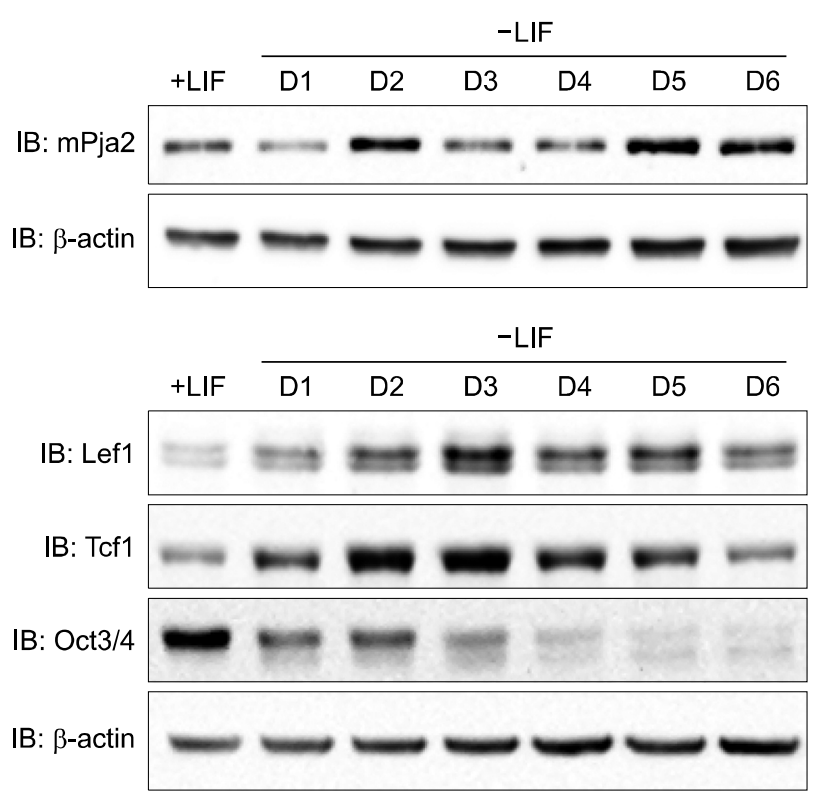

Fig. 3. Inverse correlation between expression of pja2 and TCF/LEF1. Samples were collected from undifferentiated and differentiated $\mathrm{mESCs}$ on the indicated days in the figure, and Western blotting was performed with the indicated antibodies. Differentiation of mESCs was induced by removal of LIF from culture media.

with HA-tagged TCF1 and LEF1 into HEK293T cells along with Flag-tagged Ub. Lysates were immunoprecipitated with anti-HA antibody and immunoblotted with anti-Flag antibody. As shown in Figure 4C and $4 \mathrm{D}, \mathrm{TCF} 1$ and LEF1 were more ubiquitinated by pja2 (Fig. 4C, D). Although further study is needed to verify that the level of TCF/LEF1 is directly down-regulated by pja2 during mESC differentiation, our current data indicate that pja2 interacted with and down-regulated TCF/LEF1 by enhancing ubiquitination.

\section{Discussion}

Embryonic stem cells can self-renew and differentiate into cells of three germ layers, including ectoderm, mesoderm, and endoderm. Discovery of proper controlling procedures for self-renewal and differentiation is a major goal for stem cell researchers. The lists of proteins expressed in undifferentiated and differentiated embryonic stem cells are obviously different. These differences are achieved by the regulation of differential gene expression at the transcriptional level as well as regulation of protein stability at the post-transcriptional level. Interestingly, ubiquitination levels of proteins dramatically increase during the transition from undifferentiated to differentiated mESCs (unpublished data). We propose that the rapid removal of undifferentiated stem cell-specific proteins might be mediated by the ubiquitin-proteasome pathway. Therefore, we searched for E3 ligases whose levels increased during differentiation by using microarray databases open to the public. Multiple candidates such as pja2 were identified.

Levels of both pja2 mRNA and protein increased during differentiation of mESCs (Fig. 1). Our findings suggest that the E3 ligase pja2 definitely interacted with TCF/LEF1 and regulated their stability through ubiquitin E3 ligase activity. Since Wnt/ $\beta$-catenin signaling is important for the regulation of stem cell differentiation, we 
A $\begin{array}{rlllll}\text { MYC-Pja2 } & - & + & + & + & + \\ \text { HA-TCF/Lef1 } & - & - & \text { Lef1 } & \text { Tcf1 } & \text { Tcf3 }\end{array}$

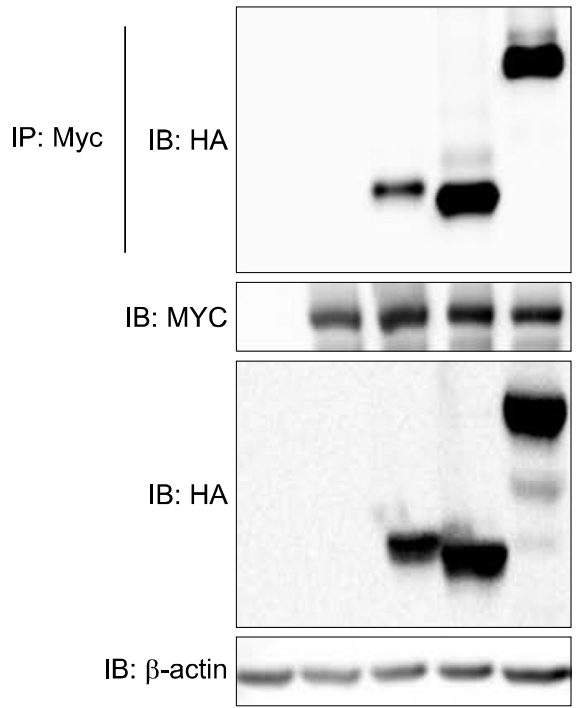

C

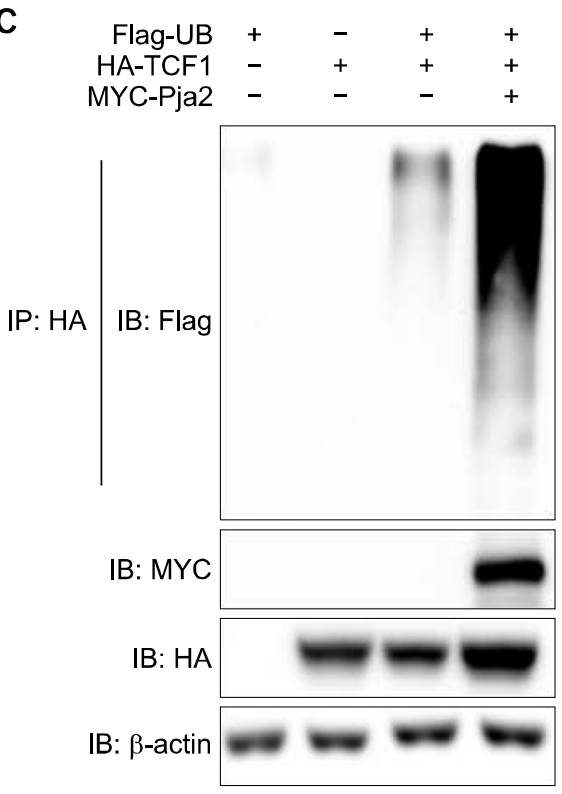

B

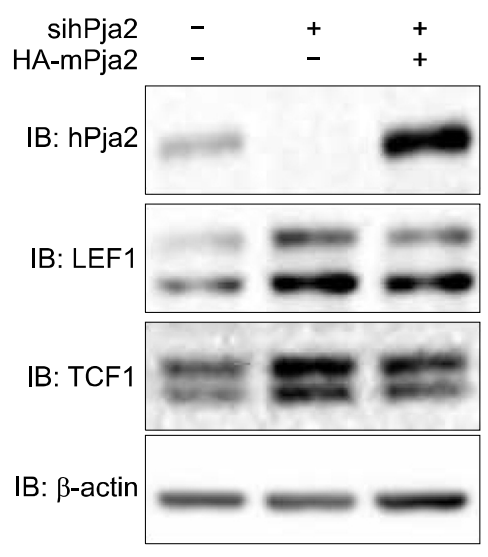

D

$\begin{array}{rrrrr}\text { Flag-UB } & + & - & + & + \\ \text { HA-LEF1 } & - & + & + & + \\ \text { MYC-Pja2 } & - & - & - & +\end{array}$

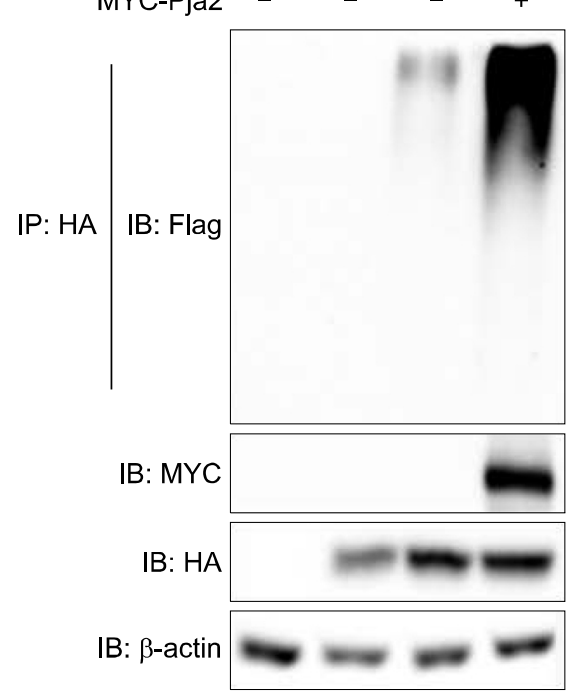

Fig. 4. Pja2 interacts and down-regulates TCF/LEF1 by enhancing ubiquitination. (A) Pja2 interacts with TCF/LEF1. MYC-pja2- and HA-tagged LEF1, TCF1, and TCF3 were co-transfected into HEK293T cells. Lysates were immunoprecipitated with anti-MYC and immunoblotted by HA. (B) Pja2 reduces levels of TCF1 and LEF1. HEK293T cells were transiently transfected with siRNAs for pja2 and HA-pja2, which is resistant to siRNA, as indicated in the figure. Expression levels of proteins were measured by immunoblotting with the antibodies indicated in the figure. (C, D) Pja2 ubiquitinates TCF/LEF1. Flag-UB, HA-TCF1/LEF1, and MYC-pja2 were transfected into HEK293T cells. Lysates were immunoprecipitated with anti-HA antibody and blotted with anti-Flag antibody to measure ubiquitination of TCF (C) and LEF1 (D). Western blotting was performed with the antibodies indicated in the figure. tested whether or not pja2 could regulate Wnt/ $\beta$-catenin signaling. Our data show that pja2 inhibited Wnt/ $\beta$ -catenin signaling by reducing the levels of TCF/LEF1. Interestingly, we observed an inverse correlation between the levels of pja2 and TCF/LEF1. Although further study is needed, our data suggest that $\mathrm{Wnt} / \beta$-catenin signaling may be regulated during ESC differentiation by the E3 ligase activity of pja2 on TCF/LEF1. Pja2 also regulates Hippo signaling by reducing the level of mobl, which resulting in increased YAP/TAZ-mediated expression of target genes involved in proliferation and anti-apoptosis (12). Therefore, increased pja2 activity during differentiation of
mESCs could lead to inhibition of Wnt/ $\beta$-catenin signaling and activation of YAP/TAZ signaling, resulting in opposite effects when only considering the roles of these signaling pathways in the regulation of proliferation. However, as YAP was shown to be necessary for the differentiation of mESCs (20), it would be interesting to test whether or not pja2 controls differentiation of mESCs by regulation of both Wnt/ $\beta$-catenin and Hippo signaling.

\section{Acknowledgments}

This research was supported by the 2017 Research Fund of the University of Seoul to E. Jho. 


\section{Potential Conflict of Interest}

The authors have no conflicting financial interest.

\section{References}

1. Murry CE, Keller G. Differentiation of embryonic stem cells to clinically relevant populations: lessons from embryonic development. Cell 2008;132:661-680

2. Doetschman TC, Eistetter H, Katz M, Schmidt W, Kemler R. The in vitro development of blastocyst-derived embryonic stem cell lines: formation of visceral yolk sac, blood islands and myocardium. J Embryol Exp Morphol 1985;87:27-45

3. Ying QL, Nichols J, Chambers I, Smith A. BMP induction of Id proteins suppresses differentiation and sustains embryonic stem cell self-renewal in collaboration with STAT3. Cell 2003;115:281-292

4. Kim H, Kim S, Song Y, Kim W, Ying QL, Jho EH. Dual Function of Wnt Signaling during Neuronal Differentiation of Mouse Embryonic Stem Cells. Stem Cells Int 2015 doi: $10.1155 / 2015 / 459301$

5. Ying QL, Wray J, Nichols J, Batlle-Morera L, Doble B, Woodgett J, Cohen P, Smith A. The ground state of embryonic stem cell self-renewal. Nature 2008;453:519-523

6. Sato N, Meijer L, Skaltsounis L, Greengard P, Brivanlou $\mathrm{AH}$. Maintenance of pluripotency in human and mouse embryonic stem cells through activation of Wnt signaling by a pharmacological GSK-3-specific inhibitor. Nat Med 2004; 10:55-63

7. Smith AG, Heath JK, Donaldson DD, Wong GG, Moreau J, Stahl M, Rogers D. Inhibition of pluripotential embryonic stem cell differentiation by purified polypeptides. Nature 1988;336:688-690

8. Ciechanover A. Proteolysis: from the lysosome to ubiquitin and the proteasome. Nat Rev Mol Cell Biol 2005;6:79-87

9. Nakayama M, Miyake T, Gahara Y, Ohara O, Kitamura T. A novel RING-H2 motif protein downregulated by axotomy: its characteristic localization at the postsynaptic density of axosomatic synapse. J Neurosci 1995;15:52385248

10. Yu P, Chen Y, Tagle DA, Cai T. PJAl, encoding a RING-H2 finger ubiquitin ligase, is a novel human $\mathrm{X}$ chro- mosome gene abundantly expressed in brain. Genomics 2002;79:869-874

11. Lignitto L, Carlucci A, Sepe M, Stefan E, Cuomo O, Nisticò R, Scorziello A, Savoia C, Garbi C, Annunziato L, Feliciello A. Control of PKA stability and signalling by the RING ligase praja2. Nat Cell Biol 2011;13:412-422

12. Lignitto L, Arcella A, Sepe M, Rinaldi L, Delle Donne R, Gallo A, Stefan E, Bachmann VA, Oliva MA, Tiziana Storlazzi C, L'Abbate A, Brunetti A, Gargiulo S, Gramanzini M, Insabato L, Garbi C, Gottesman ME, Feliciello A. Proteolysis of MOB1 by the ubiquitin ligase praja2 attenuates Hippo signalling and supports glioblastoma growth. Nat Commun 2013;4:1822

13. Nusse R. Wnt signaling and stem cell control. Cell Res 2008;18:523-527

14. ten Berge D, Kurek D, Blauwkamp T, Koole W, Maas A, Eroglu E, Siu RK, Nusse R. Embryonic stem cells require Wht proteins to prevent differentiation to epiblast stem cells. Nat Cell Biol 2011;13:1070-1075

15. Lyashenko N, Winter M, Migliorini D, Biechele T, Moon RT, Hartmann C. Differential requirement for the dual functions of $\beta$-catenin in embryonic stem cell self-renewal and germ layer formation. Nat Cell Biol 2011;13:753-761

16. Roose J, Huls G, van Beest M, Moerer P, van der Horn $\mathrm{K}$, Goldschmeding R, Logtenberg T, Clevers H. Synergy between tumor suppressor APC and the beta-catenin-Tcf4 target Tcfl. Science 1999;285:1923-1926

17. Ishitani T, Ninomiya-Tsuji J, Nagai S, Nishita $M$, Meneghini $M$, Barker $\mathrm{N}$, Waterman $\mathrm{M}$, Bowerman $\mathrm{B}$, Clevers $\mathrm{H}$, Shibuya H, Matsumoto K. The TAK1- NLK-MAPK-related pathway antagonizes signalling between beta-catenin and transcription factor TCF. Nature 1999;399:798-802

18. Li TW, Ting JH, Yokoyama NN, Bernstein A, van de Wetering M, Waterman ML. Wnt activation and alternative promoter repression of LEF1 in colon cancer. Mol Cell Biol 2006;26:5284-5299

19. Cadigan KM, Waterman ML. TCF/LEFs and Wnt signaling in the nucleus. Cold Spring Harb Perspect Biol 2012;4:a007906 doi: 10.1101/cshperspect.a007906

20. Chung H, Lee BK, Uprety N, Shen W, Lee J, Kim J. Yapl is dispensable for self-renewal but required for proper differentiation of mouse embryonic stem (ES) cells. EMBO Rep 2016;17:519-529 